\title{
Safety and effectiveness of dual guidance with video-laryngoscopy and ultrasound in jejunal tube placement in patients undergoing invasive mechanical ventilation
}

\author{
$\mathrm{Na} \mathrm{Wu}{ }^{1}$, Haitao Shen ${ }^{2} \wedge$ \\ ${ }^{1}$ Department of Endocrinology, Shengjing Hospital of China Medical University, Shenyang, China; ${ }^{2}$ Department of Emergency Medicine, Shenging \\ Hospital of China Medical University, Shenyang, China \\ Contributions: (I) Conception and design: H Shen; (II) Administrative support: H Shen; (III) Provision of study materials or patients: H Shen; (IV) \\ Collection and assembly of data: N Wu; (V) Data analysis and interpretation: N Wu; (VI) Manuscript writing: All authors; (VII) Final approval of \\ manuscript: All authors. \\ Correspondence to: Haitao Shen. Department of Emergency Medicine, Shengjing Hospital of China Medical University, no. 36, Sanhao Street, Heping \\ District, Shenyang 110004, China. Email: shenht@sj-hospital.org.
}

\begin{abstract}
Background: Appropriate and effective post-pyloric enteral nutrition (EN) is valuable for patients undergoing invasive ventilation, but blind bedside insertion of jejunal tubes is often difficult during mechanical ventilation. Here, we first report on the safety and effectiveness of inserting the jejunal tubes under the dual guidance (DG) of video-laryngoscopy and ultrasound in patients in whom jejunal tube placement during invasive mechanical ventilation is difficult.

Methods: Twenty patients in the emergency department-based intensive care unit (ICU) who were receiving invasive mechanical ventilation and had difficulty in jejunal tube placement were selected for video-laryngoscopy and ultrasound-guided jejunal tube placement (the DG group), and the outcomes were compared with patients experiencing difficult intubation before the introduction of the DG technique (the control group) in terms of the incidence of postintubation complications and the effectiveness and efficiency of nutritional support.
\end{abstract}

Results: The DG group had a significantly higher success rate of intubation and better nutritional status than the control group. The incidences of nasopharyngeal bleeding, dental and gingival injuries, and tracheostomy tube dislodgement were not significantly increased in the DG group, and the blood pressure and heart rate also showed no significant fluctuations.

Conclusions: DG with both video-laryngoscopy and ultrasound is a safe and effective technique in patients with difficulty in jejunal tube placement.

Keywords: Jejunal tube; ultrasound guidance; video-laryngoscopy; nutritional support; mechanical ventilation

Submitted Dec 24, 2020. Accepted for publication Mar 03, 2021.

doi: 10.21037/apm-21-139

View this article at: http://dx.doi.org/10.21037/apm-21-139

\section{Introduction}

Invasive mechanical ventilation is the most common life support measure for critically ill patients (1). However, patients undergoing mechanical ventilation cannot eat through the mouth, and therefore enteral nutrition (EN) or parenteral nutrition (PN) is applied. Since $\mathrm{PN}$ is expensive and has a high complication rate, $\mathrm{EN}$ is the more economic and safer option. During the implementation of EN, post-pyloric enteral feeding through a nasojejunal tube is

\footnotetext{
^ ORCID: 0000-0002-0533-0872.
} 
Table 1 General information

\begin{tabular}{lcccc}
\hline Group & $\mathrm{n}$ & Males & APACHE II & Average age \\
\hline Control group & 20 & 7 & $27.6 \pm 10.3$ & $55.5 \pm 12.3$ \\
DG group & 20 & 8 & $30 \pm 12.9$ & $58.2 \pm 12.4$ \\
\hline
\end{tabular}

widely recommended because it is associated with reduced gastroesophageal reflux and aspiration and increased caloric delivery $(2,3)$. The success rate of the traditional blind bedside jejunal tube placement method is relatively low, as the original placement route may change due to the spaceoccupying effect of the tube and the nasopharyngeal edema after tracheal intubation or from problems stemming from the tube traveling through the pylorus (4). In our center, a dual guidance (DG) method using both video-laryngoscopy and bedside ultrasound has been applied for nasojejunal tube placement which greatly increases the success rate of tube placement and effectively improves the nutritional status of the patients. To evaluate the safety and effectiveness of this technique, patients receiving our DG method (DG group) were compared with patients experiencing difficult intubation before the introduction of the DG technique (the control group) in terms of the incidence of postintubation complications and the effectiveness and efficiency of nutritional support. We present the following article in accordance with the TREND reporting checklist (available at http://dx.doi.org/10.21037/apm-21-139).

\section{Methods}

\section{General data}

The clinical data of patients who were admitted to the emergency department-based intensive care unit (ICU) of our hospital from June 2018 to October 2020 and who required jejunal tube placement during invasive mechanical ventilation were retrospectively analyzed. Placement of jejunal tubes were attempted in some patients using the blind insertion method (5). A difficult intubation was defined as five consecutive failed intubation attempts. Failed attempts were considered to occur when the tube could not be inserted further than $60 \mathrm{~cm}$; when gas could be aspired or the aspired liquid had a $\mathrm{pH}$ value of $<6$ when the tube was inserted for $110 \mathrm{~cm}$; and when post-pyloric placement was not visible on bedside plain abdominal X-ray. Twenty patients experiencing difficult intubation during a period from 2018 to 2019 were enrolled as the control group; 20 patients experiencing difficult intubation in 2020 and who received jejunal tube placement under the dual guidance of both video-laryngoscopy and ultrasound were enrolled as the DG group. Patients with a duration of mechanical ventilation less than 3 days were excluded. The general data of these patients are shown in Table 1. The study was approved by the ethics committee of our hospital (IRB number: 2020PS027J). All procedures performed in this study involving human participants were in accordance with the Declaration of Helsinki (as revised in 2013). Because of the retrospective nature of the research, the requirement for informed consent was waived.

\section{Materials and equipment}

The following materials and equipment were used for all procedures: a VLS-C2 portable anesthesia video laryngoscope (Yi'ailu Medical Equipment Company, China), a Biosound MyLab 30CV bedside ultrasound machine (Esaote Group, Italy), Flocare spiral jejunal tube (Nutricia, the Netherlands), lidocaine aerosol (Sinopharm, China), and $\mathrm{pH}$ test paper (Sichuan Science Ltd., China) Other devices included a 50-ml syringe and paraffin wax.

\section{Procedures}

First, the dental pad was removed, and lidocaine aerosol was sprayed twice into the oropharynx for surface anesthesia on the pharynx. Guidance was initiated 3 minutes after the spray. Operator A stood at the head side of the patient and gently placed the laryngoscope through a corner of the mouth, during which pull-off of the tracheal tube was avoided. The larynx was fully exposed under the laryngoscope. Any local secretion was promptly removed to ensure that the laryngeal structure was clearly visible on the laryngoscope screen. The jejunal tube was inserted through the nose. Under the direct view of the pharyngoscope, the tip of the jejunal tube was slowly pushed into the larynx and then introduced into the esophageal orifice (Figure 1A), during which any injury to the glottis was avoided. Subsequently, operator A continued to gently push the 

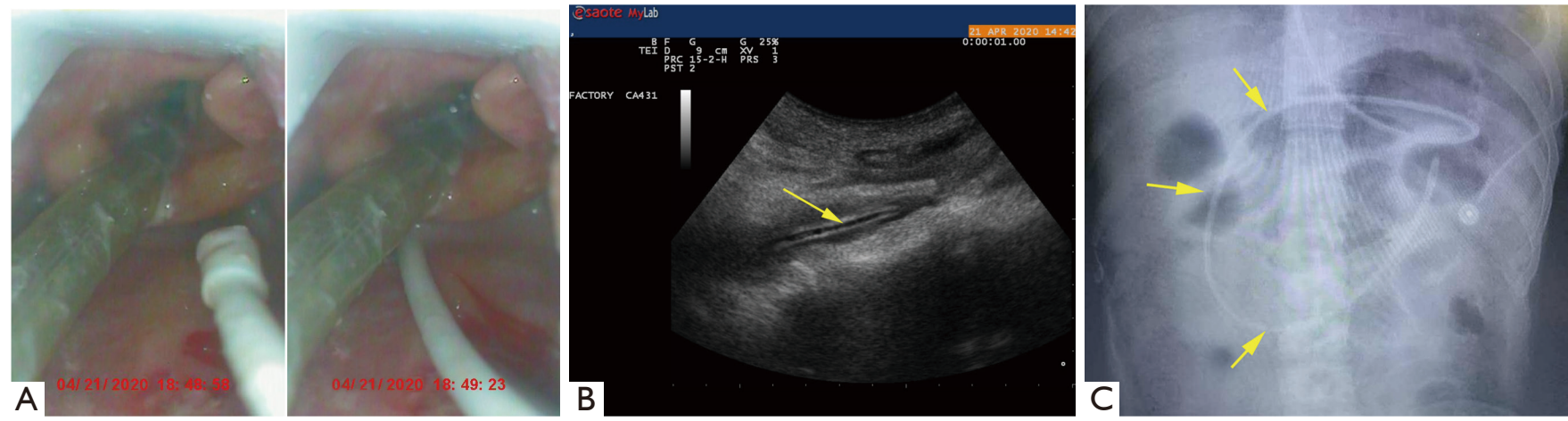

Figure 1 The process of guidance. (A) Insertion of the jejunal tube into the esophagus under the guidance of anesthesia video laryngoscope. (B) Entry of the nasojejunal tube into the jejunum under the guidance of ultrasound (the arrow shows the double-track sign of the jejunal tube under ultrasound). (C) Confirmation of position of the jejunal tube using plain abdominal X-ray (the arrows show the placement of the jejunal tube into the duodenal segment).

jejunal tube forwards until a distance of about $30 \mathrm{~cm}$ was reached. Operator B performed neck ultrasound to confirm that the jejunal tube had entered the esophagus. Then, when about $50 \mathrm{~cm}$ of the jejunal tube had been inserted, it was passed through the cardia. Next, $300 \mathrm{~mL}$ of warm water was injected, and the tube passed through the body of the stomach when the insertion length was about $55 \mathrm{~cm}$, during which "double track sign" or "snowstorm sign" was visible with ultrasound. The pharyngoscope was then withdrawn. Operator A continued to gently push the tube forward until it passed through the gastric sinus (65 $\mathrm{cm}$ in length). A feeling of emptying indicated that the tube had passed through the pylorus. The tube entered the jejunum when the inserted length was about $110 \mathrm{~cm}$, during which the aspirated fluid had a $\mathrm{pH}$ value of $>6$. Ultrasound was used to ensure that there were no more than two double-track signs (indicating the jejunal tube was folded) in the stomach, and the double-track sign of the jejunal tube could be seen in the pyloric canal (Figure 1B). The guidewire was slowly withdrawn, and its distal end was closed and then fixed caudally. Bedside plain abdominal $\mathrm{X}$-ray was performed to observe whether the jejunal tube was in place (Figure 1C). All steps of the procedure were performed gently to avoid gastrointestinal tract injury or cardiovascular stress due to excessive stimulation.

\section{Main measures}

The main measures included the following: (I) whether or not the intubation was successful, which was determined by bedside abdominal $\mathrm{X}$-ray after the tube was placed behind the pylorus; (II) local injuries, including nasal/ oropharyngeal hemorrhage, tooth and gum injuries, and/or tracheostomy tube dislodgement; (III) fluctuation of blood pressure, with the average real variability (ARV) of the mean arterial pressure continuously monitored every 3 minutes after the operation being used for comparison; (IV) heart rate variability (HRV), with the absolute value (maximum heart rate during intubation-heart rate before intubation) being used for comparison; and (V) the degree of nutritional decline (serum pre-albumin before tracheal intubationserum pre-albumin 3 days after tracheal intubation).

\section{Statistical methods}

Statistical analysis was performed using SPSS 13.0 software package (IBM Corp., USA). Numeration data were compared using chi-square test. The $t$ test was used for the analysis of normally distributed data (including age, $\mathrm{ARV}$, and prealbumin decline), and the rank-sum test was performed for nonnormally distributed data (including Apache II score and HRV). Factors influencing the success rate were analyzed using the logistic regression model. A P value of $<0.05$ was considered significantly different.

\section{Results}

\section{Success rate of intubation}

In the control group, the intubation succeeded in 3 cases and failed in 17 cases; in the DG group, the intubation succeeded in 19 cases and failed in 1 case. The success rate 
Table 2 Success rate of intubation

\begin{tabular}{lccccc}
\hline Group & Success & Failure & $\chi^{2}$ & P value & Success rate \\
\hline Control group & 3 & 17 & 25.86 & $<0.001$ & $15 \%$ \\
DG group & 19 & 1 & & $95 \%$ & \\
\hline
\end{tabular}

Table 3 Binary logistic regression analysis of factors including the success rate of intubation

\begin{tabular}{lccc}
\hline Factors & Regression coefficient & OR & P value \\
\hline Guidance & 4.68 & 107.7 & $<0.001$ \\
\hline
\end{tabular}

OR, odds ratio.

significantly differed between these two groups (Table 2). Binary logistic regression analysis with factors including application of guidance, age, sex, APACHE II score, oropharyngeal injury, ARV, and HRV showed that guidance significantly increased the success rate of intubation (Table 3).

\section{Evaluation of the parameters}

The oropharyngeal injury, ARV, HRV, and degree of nutritional decline in the two groups are shown in Table 4. Although the incidence of oropharyngeal injury, ARV, and HRV were slightly higher in the DG group, the differences were not statistically significant; notably, the maintenance of nutritional status in the DG group was superior to that in the control group.

\section{Discussion}

Invasive mechanical ventilation is the most effective lifesaving measure for respiratory failure (1), and the number of patients receiving mechanical ventilation is increasing due to various factors such as the aging population and the 2019 novel coronavirus (COVID-19) pandemic (6,7). While patients undergoing invasive mechanical ventilation often experience increased energy expenditure, they are typically in a state of inadequate caloric supply due to the inability to swallow. Therefore, adequate clinical nutritional support is essential for these patients. In clinical settings, the jejunal tube has become an important part of EN provision in mechanically ventilated patients, as it can offer a stable amount of nutrition with mild side effects $(8,9)$. However, placing a jejunostomy tube in patients with invasive mechanical ventilation may be particularly challenging.
First, the tracheal tube currently used in clinical practice is a disposable tube with its own inflatable sleeve, and the sleeve is routinely inflated after successful tracheal intubation to close the airway gap and ensure the effectiveness of ventilation; unfortunately, the inflatable sleeve also causes external pressure on the adjacent esophagus, and the local mucosal congestion and edema of the trachea after tracheal tube placement can cause narrowing of the esophageal inlet, which can cause difficulties in the passage of the jejunal tube through the pharynx. Critically ill patients who are mechanically ventilated often have an altered state of consciousness and/ or dull or absent reflexes, and they cannot mimic swallowing while the tube is being placed, which makes it more difficult for the jejunal tube to pass through the pharynx. As a result, the jejunal tube may bend or coil above the entrance to the esophagus and thus cannot be advanced into the esophagus. In the past, the blind insertion method was often used for difficult placement, with the tube being repeatedly inserted, which often damaged the nasopharyngeal mucosa, causing local bleeding and infection; furthermore, the edema compressing the tracheal tube could also be fatal.

The video laryngoscope is a laryngoscope with a screen, which increases the visual field during intubation (10). During the insertion of the jejunal tube, the throat structures are always in the operator's visual field, which can effectively reduce the throat injury caused by blind intubation. In addition, the angle and force of tube insertion can be adjusted in real-time, which can improve the success rate and safety of the jejunal tube passage through the throat. Our current study also demonstrated that videolaryngoscopy can significantly increase the success rate of intubation without additional injury.

Second, the success rate of jejunal tube insertion through the pylorus into the duodenum is particularly low and depends highly on the operator's skill, as this step cannot be observed directly and can only be inserted blindly by hand $(11,12)$. Although the success rate of jejunal tube placement into the duodenum has been increased with the implementation of interventional guidance or endoscopic guidance (13), these techniques are not 
Table 4 Comparisons of risk-related parameters

\begin{tabular}{lcccc}
\hline Group & Oropharyngeal injury & ARV & HRV & Prealbumin decline \\
\hline Control group & 3 & $10.6 \pm 6.9$ & $21.5 \pm 11$ & $0.68 \pm 0.49^{\star}$ \\
DG group & 6 & $12.8 \pm 7.1$ & $23.5 \pm 12.3$ & $0.21 \pm 0.54^{\star}$ \\
\hline
\end{tabular}

${ }^{*} \mathrm{P}<0.05$. ARV, average real variability; HRV, heart rate variability.

feasible for mechanically ventilated patients in the ICU. Even worse, mechanically ventilated COVID-19 patients require isolation (7), which further increases the difficulty of interventional or endoscopic guidance. Thus, bedside ultrasound without leaving the isolation setting becomes the optimal option for guided tube placement. In our study, ultrasound-guided jejunal tube placement enabled the direct observation of the echoes inside the intraduodenal canal and thus guided the placement process, which significantly increased the success rate of jejunal tube placement.

Mean arterial pressure is a good hemodynamic parameter and thus can be used to assess surgical risk (14). In recent years, numerous studies have used mean arterial pressure variations such as time-weighted mean arterial pressure (TWA) and ARV as prognostic indicators $(15,16)$. Our current study was a retrospective analysis, in which the TWA calculated from continuous waveforms was not available; therefore, we chose ARV, which can be collected retrospectively, as the indicator for assessing surgical risks.

The purpose of jejunal tube placement is to maintain a normal nutritional status, so we also compared the nutritional status of patients in the two groups. Typically, scoring systems such as the Mini Nutritional AssessmentShort Form (MNA-SF) and Nutritional Risk Screening 2002 (NRS-2002) are used for nutritional status assessment (17); however, they are not feasible for patients with acute diseases. Changes in nutritional status can be assessed using simple and easy indicators such as weight or body max index in healthy or mildly ill patients (18); in critically ill patients, however, conditions such as edema and dehydration occur frequently and can directly affect these indicators (19). Therefore, our study employed serum prealbumin levels, which have the highest value for nutritional assessment in critically ill patients, to evaluate nutritional status $(20,21)$. Our data also showed that the nutritional status of patients in the DG group was significantly better than that of patients in the control group. DG intubation can cope with the common difficult intubation situations encountered by patients with invasive ventilator, but some special situations such as collapsed intestine. pyloric stenosis and other special situations require special treatments (22). We also found that the DG group had higher surgical risk than the control group. Therefore, guided intubation is not recommended for all patients, and should be preferred mainly for patients in whom intubation is difficult.

Although our findings are encouraging, biases arising from the retrospective nature of the analysis were unavoidable. Therefore, a prospective controlled study with more samples is warranted to further evaluate the safety, effectiveness, and clinical feasibility of dual guidance with video-laryngoscopy and ultrasound in jejunal tube placement.

\section{Conclusions}

Dual guidance with video-laryngoscopy and ultrasound is a safe and effective technique for jejunal tube placement in mechanically ventilated patients for whom jejunal tube placement is difficult.

\section{Acknowledgments}

This work is supported by the Neusoft Research of Intelligent Healthcare Technology, Co. Ltd. We thank Neusoft and Neusoft Research of Intelligent Healthcare Technology for their support with the data extraction, analysis, and evaluation by leveraging RealOne ISCI and RealMedSci, their clinic research platform and automatic medical analysis platform.

Funding: This study was supported by funding from the Science Foundation of Liaoning Education Department (no. FWZR2020012) and the 345 Talent Project of Sheng Jing Hospital.

\section{Footnote}

Reporting Checklist: The authors have completed the TREND reporting checklist. Available at http://dx.doi. org/10.21037/apm-21-139 
Data Sharing Statement: Available at http://dx.doi. org/10.21037/apm-21-139

Conflicts of Interest: Both authors have completed the ICMJE uniform disclosure form (available at http://dx.doi. org/10.21037/apm-21-139). The authors have no conflicts of interest to declare.

Ethical Statement: The authors are accountable for all aspects of the work in ensuring that questions related to the accuracy or integrity of any part of the work are appropriately investigated and resolved. All procedures performed in this study involving human participants were in accordance with the Declaration of Helsinki (as revised in 2013). The study was approved by the ethics committee of our hospital (IRB number: 2020PS027J). Because of the retrospective nature of the research, the requirement for informed consent was waived.

Open Access Statement: This is an Open Access article distributed in accordance with the Creative Commons Attribution-NonCommercial-NoDerivs 4.0 International License (CC BY-NC-ND 4.0), which permits the noncommercial replication and distribution of the article with the strict proviso that no changes or edits are made and the original work is properly cited (including links to both the formal publication through the relevant DOI and the license). See: https://creativecommons.org/licenses/by-nc-nd/4.0/.

\section{References}

1. Pham T, Brochard LJ, Slutsky AS. Mechanical Ventilation: State of the Art. Mayo Clin Proc 2017;92:1382-400.

2. Kreymann KG, Berger MM, Deutz NE, et al. ESPEN Guidelines on Enteral Nutrition: Intensive care. Clin Nutr 2006;25:210-23.

3. Villet $\mathrm{S}$, Chiolero RL, Bollmann MD, et al. Negative impact of hypocaloric feeding and energy balance on clinical outcome in ICU patients. Clin Nutr 2005;24:502-9.

4. Wan B, Fu H, Yin J. Early jejunal feeding by bedside placement of a nasointestinal tube significantly improves nutritional status and reduces complications in critically ill patients versus enteral nutrition by a nasogastric tube. Asia Pac J Clin Nutr 2015;24:51-7.

5. Duggan S, Egan SM, Smyth ND, et al. Blind bedside insertion of small bowel feeding tubes. Ir J Med Sci 2009;178:485-9.
6. Halpern NA, Goldman DA, Tan KS, et al. Trends in Critical Care Beds and Use Among Population Groups and Medicare and Medicaid Beneficiaries in the United States: 2000-2010. Crit Care Med 2016;44:1490-9.

7. Simonnet A, Chetboun M, Poissy J, et al. High Prevalence of Obesity in Severe Acute Respiratory Syndrome Coronavirus-2 (SARS-CoV-2) Requiring Invasive Mechanical Ventilation. Obesity (Silver Spring) 2020;28:1195-9.

8. McClave SA, Martindale RG, Vanek VW, et al. Guidelines for the Provision and Assessment of Nutrition Support Therapy in the Adult Critically Ill Patient: Society of Critical Care Medicine (SCCM) and American Society for Parenteral and Enteral Nutrition (A.S.P.E.N.). JPEN J Parenter Enteral Nutr 2009;33:277-316.

9. Heyland DK, Dhaliwal R, Drover JW, et al. Canadian clinical practice guidelines for nutrition support in mechanically ventilated, critically ill adult patients. JPEN J Parenter Enteral Nutr 2003;27:355-73.

10. Russell TM, Hormis A; Rotherham NHS Foundation Trust. Should the Glidescope video laryngoscope be used first line for all oral intubations or only in those with a difficult airway? A review of current literature. J Perioper Pract 2018;28:322-33.

11. Puiggròs C, Molinos R, Ortiz MD, et al. Experience in Bedside Placement, Clinical Validity, and Cost-Efficacy of a Self-Propelled Nasojejunal Feeding Tube. Nutr Clin Pract 2015;30:815-23.

12. Hernández-Socorro CR, Marin J, Ruiz-Santana S, et al. Bedside sonographic-guided versus blind nasoenteric feeding tube placement in critically ill patients. Crit Care Med 1996;24:1690-4.

13. Reed RL 2nd, Eachempati SR, Russell MK, et al. Endoscopic placement of jejunal feeding catheters in critically ill patients by a "push" technique. J Trauma 1998;45:388-93.

14. Salmasi V, Maheshwari K, Yang D, et al. Relationship between Intraoperative Hypotension, Defined by Either Reduction from Baseline or Absolute Thresholds, and Acute Kidney and Myocardial Injury after Noncardiac Surgery: A Retrospective Cohort Analysis. Anesthesiology 2017;126:47-65.

15. Ahuja S, Mascha EJ, Yang D, et al. Associations of Intraoperative Radial Arterial Systolic, Diastolic, Mean, and Pulse Pressures with Myocardial and Acute Kidney Injury after Noncardiac Surgery: A Retrospective Cohort Analysis. Anesthesiology 2020;132:291-306.

16. Patidar KR, Peng JL, Pike F, et al. Associations Between 
Mean Arterial Pressure and Poor ICU Outcomes in Critically Ill Patients With Cirrhosis: Is 65 The Sweet Spot? Crit Care Med 2020;48:e753-60.

17. Helminen H, Luukkaala T, Saarnio J, et al. Predictive value of the mini-nutritional assessment short form (MNA-SF) and nutritional risk screening (NRS2002) in hip fracture. Eur J Clin Nutr 2019;73:112-20.

18. Silva APD, Feilbelmann TCM, Silva DC, et al. Prevalence of overweight and obesity and associated factors in school children and adolescents in a medium-sized Brazilian city. Clinics (Sao Paulo) 2018;73:e438.

19. Vincent JL. Fluid management in the critically ill. Kidney Int 2019;96:52-7.

Cite this article as: $\mathrm{Wu} \mathrm{N}$, Shen H. Safety and effectiveness of dual guidance with video-laryngoscopy and ultrasound in jejunal tube placement in patients undergoing invasive mechanical ventilation. Ann Palliat Med 2021;10(3):3128-3134. doi: 10.21037/ apm-21-139
20. Joffe A, Anton N, Lequier L, et al. Nutritional support for critically ill children. Cochrane Database Syst Rev 2016;2016:CD005144.

21. Keller U. Nutritional Laboratory Markers in Malnutrition. J Clin Med 2019;8:775.

22. Sasaki T, Mie T, Takeda T, et al. Percutaneous insertion of a jejunal tube via the collapsed blind end of elevated jejunum by a balloon puncturing method assisted by a balloon enteroscope. J Hepatobiliary Pancreat Sci 2020;27:663-4.

(English Language Editor: J. Gray) 